\title{
EL ENTRENAMIENTO FíSICO MÍLITAR ES UN ENTRENAMIENTO DE ALTO RENDIMIENTO. UNA EXPERIENCIA DE INVESTIGACIÓN EN LA ESCUELA MILITAR DE CADETES GENERAL JOSE MARIA CORDOVA ${ }^{1}$
}

\author{
Laura Elizabeth Castro Jiménez² \\ Paula Janyn Melo Buitrago ${ }^{3}$
}

\section{RESUMEN}

El entrenamiento físico militar, conlleva a unas altas exigencias físicas en los sujetos por el tiempo de concentración, por las horas de entrenamiento y la densidad de las actividades. Es decir se puede considerar a esta población como de alto rendimiento por que duran entre 6 meses y más con entrenamiento físico militar diario, por lo que se encuentra asociado, inevitablemente, con la aparición de lesiones, las cuales pueden llevar a procesos de rehabilitación demorados y truncar la vida militar de las personas y generar fallas en la seguridad del país (Olmedilla Zafra, Ortega Toro, \& Prieto Andréu, 2009) (Heinrich, Spencer, Fehl, \& Poston, 2012). El objetivo de la investigación es conocer las lesiones producto del entrenamiento físico militar en la Escuela Militar de Cadetes General José María Córdova. Metodología. Este estudio es de tipo cuantitativo, de corte descriptivo. Se diseñó una encuesta para conocer las lesiones producto del entrenamiento físico militar. Resultados. Dentro de los resultados se evidencia que muchas de lesiones presentadas son por sobre-entrenamiento y sobreesfuerzo, por parte de los militares al mando o de los mismos cadetes, motivados por sobresalir, tener mejores condiciones al interior de la Escuela o por castigos que en su mayoría son ejercicios físicos por acciones en contra de su reglamento. Conclusiones. Esta es una población que generalmente no se toma en cuenta en el área de la Educación Física por ser la fuerza que protege al país y se desconoce el contexto en que se deben entrenar y las consecuencias de ellos para salir a brindar un servicio al país.

Palabras claves: Entrenamiento, alto rendimiento, militares

\section{ABSTRACT.}

Military physical training, leads to a high physical demands on the subject for the time of concentration for hours training and density of activities. le you can consider this population as

\footnotetext{
${ }^{1}$ Este artículo es parte de los resultados parciales obtenidos en el proyecto de investigación titulado: "Caracterización de las lesiones en los estudiantes de la Escuela Militar de Cadetes General José María Córdova en el entrenamiento físico militar" que se viene desarrollando de manera conjunta entre las Instituciones: Universidad Santo Tomás y Escuela Militar de Cadetes, haciendo parte de las líneas de investigación del grupo Cuerpo, Sujeto y Educación y entrenamiento físico militar en el grupo FORTIUS respectivamente.

${ }^{2}$ Magister en Salud Pública de la Universidad Nacional, Docente Investigador del Grupo Cuerpo, sujeto y educación de la Facultad de Cultura Física Deporte y Recreación de la Universidad Santo Tomás. Laura.castro@usantotomas.edu.co

${ }^{3}$ Magister en Fisiología del deporte de la Universidad del Valle, Coordinadora de investigaciones de la Facultad de Educación Física Militar en la Escuela Militar de Cadetes "General José María Córdova". paulajanynmb@yahoo.es
} 
high performance for lasting between 6 months and older with physical training daily military, so it is associated inevitably with the appearance of lesions, which can lead to rehabilitation processes delayed and truncate the military lives and create security breaches in the country (Olmedilla Zafra, Ortega Toro Prieto \& Andreu, 2009) (Heinrich, Spencer, Fehl, \& Poston, 2012). The objective of the research is to know the product injury of military physical training in General José María Córdova Military Cadet School. Methodology. This study is quantitative, descriptive court. A survey to find the product injury of military physical training was designed. Results. Among the results are evidence that many lesions presented are overtraining and overexertion, by military command or thereof cadets motivated to excel, have better conditions within the School or punishment in its most exercises are physical actions against its rules. Conclusions. This is a population that is generally not taken into account in the area of Physical Education to be the force that protects the country and the context in which to train and the consequences of them are known to go to providing a service to the country.

KEY WORDS: Training, high performance military

\section{INTRODUCCIÓN}

Todos los países tienen como base para ejercer su soberanía la conformación de ejércitos, los cuales teniendo como objetivo "defender la soberanía, la independencia y la integridad territorial, proteger a la población civil, los recursos privados y estatales para contribuir a generar un ambiente de paz, seguridad y desarrollo, que garantice el orden constitucional de la nación" (EJERCITO NACIONAL DE COLOMBIA, 2014), es así, como la "Escuela Militar de Cadetes General José María Córdova, alma mater del Ejército Nacional es la encargada de formar integralmente a los futuros oficiales del Ejército como líderes comandantes de pelotón, profesionales en ciencias militares y otras disciplinas, con sólidas competencias fundamentales en principios y valores institucionales, en función del desarrollo y la seguridad nacional" (EJERCITO NACIONAL DE COLOMBIA, 2014).

De esta manera, la formación académico-militar de Colombia, reciben un entrenamiento militar durante un período de cuatro años en los cuales cursan paralelamente estudios universitarios de pregrado en diversas disciplinas. Al ingresar al primer año reciben una instrucción básica militar inicial, posteriormente realizan su preparación como oficiales en la que deben realizar estudios de idiomas y estudios profesionales dentro de la institución en Ciencias militares y deben seleccionar una segunda carrera profesional entre las siguientes: Administración logística, Ingeniería Civil, Derecho, Relaciones Internacionales o Educación Física Militar.

Los cadetes de último año en la escuela reciben el grado de alférez y su ascenso se representa con una ceremonia de entrega de sables. Al finalizar su formación, el director de la Escuela entrega al Comandante General del Ejército a los nuevos oficiales, quienes reciben el título de subtenientes en una ceremonia oficial y los diplomas profesionales de las carreras universitarias realizadas durante su formación. 
Dentro de la formación física militar, se realizan sesiones 4 a 5 días a la semana, con una duración entre dos y tres horas diarias como mínimo bajo una planificación estructurada de acuerdo al nivel de preparación en el que se encuentran; aspectos que nos permiten considerarlos como deportistas de rendimiento, acercándonos al léxico deportivo, por la alta exigencia en su entrenamiento, y la práctica sistemática que se observa en sus planes de curso; toda vez que tengamos en cuenta que el entrenamiento deportivo comprende tres aspectos esenciales: la noción de rendimiento en el sentido genérico del término, la de especificidad de los ejercicios, y la noción de planificación. (Martín, 2001)

Weineck (2005) define la capacidad de rendimiento deportivo como "el grado de mejora posible de un rendimiento de orden motriz, de una actividad deportiva determinada"; es así como, el entrenamiento físico militar, en la búsqueda de lograr el mejor resultado en el campo de batalla, expone a sus hombres a altas exigencias físicas, por el tiempo de concentración, por las horas de entrenamiento, la densidad de las actividades y las pruebas de evaluación en las que miden sus capacidades. Es decir se puede considerar a esta población como de alto rendimiento, no sólo por que duran entre 6 meses y más con entrenamiento físico militar diario, sino porque con ello persiguen el desarrollo de los potenciales humanos propios de la vida militar desde los aspectos físico, psicológico y social, situación que se acerca a la definición del alto rendimiento deportivo, considerado como "la máxima expresión del desarrollo de los potenciales humanos en una práctica deportiva a máximo nivel competitivo" (Pila H, s.f.). Por lo anterior, el entrenamiento físico militar se encuentra asociado, inevitablemente, con la aparición de lesiones, las cuales pueden llevar a procesos de rehabilitación demorados y truncar la vida militar de las personas (Olmedilla Zafra, Ortega Toro, \& Prieto Andréu, 2009) (Heinrich, Spencer, Fehl, \& Poston, 2012).

Keith, en su estudio realizado en Estados Unidos, menciona que aunque las lesiones son reconocidas como un importante problema de salud en el ejército, la magnitud del problema se subestima, cuando sólo se consideran las lesiones agudas traumáticas; y los trastornos musculoesqueléticos relacionados con las lesiones son comunes en esta población joven y activa. Muchas de ellas implican daño físico causado por microtraumas (uso excesivo) en la recreación, el deporte, la formación, y el desempeño laboral. En 2006, hubo 743.547 lesiones musculoesqueléticas relacionadas (ambulatoria y hospitalaria, combinada) en militares, incluyendo los diagnósticos primarios y no primarios. En la matriz, el $82 \%$ de los trastornos musculoesqueléticos relacionados con las lesiones se clasificaron en: inflamación/dolor (uso excesivo), seguido de alteraciones articulares (15\%) y las fracturas por estrés (2\%). Articulaciones como la rodilla (22\%), la columna lumbar $(20 \%)$, y el tobillo / pie $(13 \%)$ son las zonas del cuerpo más lesionados. Concluyendo, que es necesario evaluar la magnitud del problema de las lesiones en los servicios militares, las afecciones musculoesqueléticas relacionadas con lesiones deben ser incluidos. Cuando estas lesiones se combinan con lesiones traumáticas agudas, hay casi 1,6 millones de consultas médicas relacionadas con lesiones cada año. La matriz proporciona un formato estandarizado para clasificar estas lesiones, hacer comparaciones a través del tiempo, y se centran los esfuerzos de prevención en los tipos de lesiones que conducen y / o regiones del cuerpo.(Keith, Bruce, Steven, \& Bullock) 
Castro Solano y Martina Casullo (2002), realizaron un estudio que tuvo como objetivo identificar los factores asociados con rendimientos en los aprendizajes académicos y militares exitosos y no exitosos en ingresantes y estudiantes próximos a su graduación, encontrando que los aspectos sicológicos son poco abordados, así como han sido poco tratados los relacionados con la preparación físico-militar. Castro Solano y Fernandez Lopirace (2005) analizaron seis cohortes de egresados de una institución militar (1997-2003), atendiendo a la variabilidad en el rendimiento en su educación superior, relacionándolo con variables sociodemográficas (educación y ocupación parentales y estatus de pariente de militar). Los resultados indican que los promedios generales de carrera observan una tendencia descendente en el tiempo. El rendimiento militar muestra menor variabilidad, en tanto que los rendimientos académico y militar aparecen ligados al nivel educativo parental (Lopirace, 2005)

Mohammadi et all, (2013) mencionan que la alta incidencia de lesiones en los miembros inferiores asociadas con ejercicios físicos en reclutas militares, sugiere que la fatiga puede ser un factor de riesgo de lesiones; por lo que al evaluar si los ejercicios militares podrían alterar el tobillo y la rodilla y examinar la posible relación articular de tobillo y rodilla en futuras lesiones de las extremidades inferiores en el servicio militar, se encontró interacción del grupo por tiempo de todas las variables, todos los participantes mostraron desalineación articular después del ejercicio $(p<0,01)$, pero el grupo de atletas lesionados tenían mayores cambios para todas las variables ( $P$ $<0,01$ ) (Mohammadi, Azma, Naseh, Emadifard, \& Etemadi, 2013).

A fin de valorar correctamente la incidencia de las lesiones producidas por el entrenamiento físico militar y para poder eliminar posibles perjuicios, es necesario interpretar correctamente las estadísticas de accidentes y conocer los diferentes aspectos sobre la base de los que se obtienen las cifras. A tal fin, cabe señalar que el análisis estadístico proporciona indicios esenciales para reducir el riesgo de lesiones y demuestra que la selección específica de los ejercicios, una preparación adecuada y el conocimiento de las causas de accidentes y su prevención pueden minimizar el riesgo y, por otro lado, disminuir el peligro de presentarse en patologías crónicas.

\section{METOdOLOGÍA}

Este estudio es corte cuantitativo. El diseño de investigación propuesto es descriptivo, el cual según Hernández busca especificar propiedades, características y rasgos importantes de cualquier fenómeno que se analice. Describe tendencias de un grupo o población lo cual es útil para mostrar con precisión los ángulos o dimensiones de un fenómeno, suceso, comunidad, contexto o situación (Hernandez Sampiere, 2010).

Se diseñó un formato de recolección de datos, tipo encuesta, dirigido a los cadetes de la Escuela Militar de Cadetes General José María Córdoba. A través de este se buscó indagar sobre los siguientes aspectos:

- Presencia de lesiones (número de lesiones)

- Mecanismo de lesión (directo, indirecta, agudo, crónico)

- Tipo de lesión (lesiones osteomusculares) 
Después de realizar los ajustes pertinentes al instrumento se aplicó éste a los cadetes de la Escuela Militar de Cadetes General José María Córdoba. Conseguida la información se almacenara en una base de datos para analizarla a través del programa SPSS.

El tamaño de muestra se tomó una muestra de cadetes de la Escuela Militar de Cadetes General José María Córdoba, se decidió que el muestreo se realizó empleando la siguiente fórmula de muestreo:

$$
n=\frac{N^{*} Z_{a}^{2} p^{*} q}{d^{2} *(N-1)+Z_{a}^{2} p^{*} q}
$$

La proporción esperada (p) será de 0.2, teniendo en cuenta que actualmente tenemos una población cautiva en la Escuela Militar. Tomar una proporción esperada ( $p$ ) de 0.2 lleva a la necesidad de ampliar el tamaño de la muestra el nivel de significancia será de al 0,05 (tomando un valor de confiabilidad del 95\%).

\section{RESULTADOS}

Para la descripción de los resultados se mencionara lo encontrado en los primeros 5 niveles, ya que a partir del sexto tienen entrenamiento en mando que complejiza aún más el entrenamiento físico militar. De acuerdo a los resultados de las encuestas encontramos el $64,6 \%$ de los estudiantes de primer nivel realiza un entrenamiento de dos horas mínimas de duración, el 27,1\% de 4 horas, y el $8,4 \%$ más de 5 horas diarias. De estos solo el 10,5\% entrena menos de tres días a la semana, el 35,4\% 5 días y el 54,2\% más de 6 días a la semana. Los estudiantes de segundo nivel reportan que el 41,5\% entrena 2 horas diarias, el 32,1\% 4 horas y el 22,6\% más de 5 horas; aumentando el porcentaje de entrenamiento de mayor duración con respecto al nivel anterior.

Los de tercer nivel mencionan que el 24,4\% entrena dos horas diarias, el $45,1 \% 4$ horas y el $26,9 \%$ más de 5 horas diarias; aumentando en comparación a niveles inferiores. En el cuarto nivel, la duración del entrenamiento es de 2 horas en el 50\%; 4 horas en el 20,7\% y mayor de 5 horas en el $22,4 \%$. Los de quinto nivel el 55,6 tienen un entrenamiento de 2 horas diarias, 4 horas el 25,9\% y 18,5 más de 5 horas al día. Encontrando un aumento de la duración del entrenamiento cuando se sube el nivel, exceptuando el quinto nivel que disminuye un poco.

En cuanto a los días de entrenamiento en el nivel dos se menciona que el 45,3\% entrena 5 días a la semana y el 50,9\%; más de 6 días a la semana. El nivel tres refiere que el 57,3\% entrena más de 6 días a la semana, el 23,2\% 5 días a la semana y el porcentaje restante menos de tres días. Los de cuarto nivel informan que tres días el 24,1\%; 5 días el 36,2\% y mayor de 6 días el 36,2\%. Y quinto 
nivel entrenan el 49,4\% 5 días a la semana y el 32\% más de 6 días a la semana; mostrando un mantenimiento en la frecuencia del entrenamiento.

Los cadetes de primer nivel referencian que aparte de su entrenamiento físico militar diario, realizan el promedio en el día de 90,2 flexiones de codo; 64,09 abdominales; 10,39 ejercicios en barra; 2,7 vueltas a la escuela; 2,6 vueltas a marte y 98,07 sentadillas; por castigos o por órdenes de los militares a cargo. En segundo nivel el reporte es 89,05 flexiones de codo; 65,6 abdominales; 15,9 ejercicios con barra; 3 vueltas a la escuela; 3,05 vueltas a marte y 59,5 sentadillas.

Los de tercer nivel mencionan que el promedio de flexiones de pecho son 124,4; el de abdominales de 75,7; ejercicios en barra 15,6; vueltas a la escuela militar 3,44; vueltas a marte 2 y 146,5 sentadillas por día. En el cuarto nivel refieren que flexiones de codo 73,7; 62,4 abdominales; 9 ejercicios en barra; 1 vuelta a la escuela militar y a marte y 76 sentadillas. Los de quinto nivel mencionan que hacen además de su entrenamiento diario 64,2 flexiones de codo; 63,8 abdominales; 11 ejercicios en barra, 2,9 vueltas a la escuela; 2 vueltas a marte y 47 sentadillas por día.

\section{DISCUSIÓN}

El entrenamiento de alto rendimiento requiere de una disciplina rigurosa en cuanto a la dedicación en tiempo y al cumplimiento de factores endógenos (constitución corporal, edad...) y exógenos (nutrición, condiciones ambientales...) que influyen definidamente en la obtención de resultados. De la misma manera el cumplimiento de los principios del entrenamiento deportivo al momento de planificar las cargas es fundamental a la hora de medir a rigurosidad en su ejecución; teniendo en cuenta que para desarrollar la capacidad de rendimiento en un proceso a largo plazo es necesario el planteamiento de objetivos, contenidos, medios y métodos de entrenamiento (Weineck, 2005; Wilmore \& Costill, 2001). El entrenamiento que se desarrolla con los estudiantes de la Escuela militar de cadetes, corresponde con un entrenamiento a largo plazo y posee los aspectos necesarios para el desarrollo dela capacidad de rendimiento, ya que posee objetivos claros, el contenido del entrenamiento es propio del contexto militar en cuanto a ejercicios de desarrollo general, específico y competitivo, que en el caso de ellos se realiza cuando asisten a las campañas en las unidades militares especiales, los medios se manifiestan en todos los escenarios que tienen dispuestos para el desarrollo de sus prácticas militares y los métodos se encuentran en la sistematización y planificación de las cargas de acuerdo al nivel en que se encuentran los estudiantes; de acuerdo a los resultados obtenidos, todo esto puede verse afectado por las cargas adicionales que los estudiantes manifestaron en la encuesta realizada, reflejado en grandes trabajos de fuerza potencia en brazos y piernas, que según las repeticiones referidas puede interpretarse en 15 minutos adicionales de trabajo intenso sin ningún tipo de calentamiento ni recuperación ya que corresponde a los ejercicios improvisados que realizan por orden, y la resistencia aeróbica que puede ser entre 30 minutos y una hora adicional. Todo esto se puede interpretar como características del entrenamiento militar, pero cuanto de todo esto puede estar afectado el rendimiento óptimo de los estudiantes? La aparición, presencia y permanencia de ciertas lesiones puede darnos una mirada diagnóstica, y así procurar las soluciones adecuadas. 


\section{CONCLUSIONES}

Los estudiantes de la escuela militar por el tiempo de dedicación y de exigencia pueden considerarse como deportistas de alto rendimiento, pero las cargas presentadas tanto físicas como académicas han conllevado a que sean susceptibles de presentar lesiones, las cuales no han sido consideradas por los profesionales del área y que se convierte en la actualidad una población que ha estado desprotegida frente a los métodos de entrenamiento como a la planificación de éstos. Es así como en la actualidad se convierte en un campo de intervención y de cuidado ya que son ellos los que tienen a cargo la seguridad del país y se debe mejorar las condiciones de calidad de vida y de salud de la población.

\section{Bibliografía}

Castro Solano, A. y. (2002, vol. 18, no 2 (diciembre)). Predictores del rendimiento académico y militarde cadetes argentinos. anales de psicología, 247-259.

Heinrich, K., Spencer, V., Fehl, N., \& Poston, W. (2012). Mission essential fitness: comparison of functional circuit training to traditional Army physical training for active duty military. Mil Med. 2012 Oct;177(10),, 1125-30.

Hernandez Sampiere, R. (2010). Metodología de la investigación, segunda edición . . México : Editorial Ultra.

Keith, H., Bruce, J., Steven, H., \& Bullock, D. (s.f.). Musculoskeletal injuries description of an underrecognized injury problem among military personnel. American Journal of Preventive Medicine.

Lopirace, C. S. (2005). Predictores Para la Selección de Cadetes en Instituciones Militares . PSYKHE, Vol. 14, № 1, , 17 - 30 .

Martínez Sánchez, J. (2011). Aspectos psicológicos de la supervivencia en operaciones militares. Sanid. mil. 2011; 67 (1). , 43-48.

Martín D., Dietrich. (2001) Manual de metodología del entrenamiento deportivo. Barcelona. Editorial: Paidotribo. 
Mohammadi, F., Azma, K., Naseh, I., Emadifard, R., \& Etemadi, Y. (2013). Military Exercises, Knee and Ankle Joint Position Sense, and Injury in Male Conscripts: A Pilot Study. J Athl Train. 2013 Apr 3.

Olmedilla Zafra, A., Ortega Toro, E., \& Prieto Andréu, J. M. (2009). Percepción de los tenistas respecto a los factores que pueden provocar lesiones: diferencias entre federados y no federados. Cuadernos de Psicología del Deporte Dirección General de Deportes-CARM 2009. Vol.9, núm. 2. Julio-Diciembre Facultad de Psicología ISSN: 1578-8423, 8-17.

Pila Hernández, Hermenegildo. (s.f.) Selección de talentos para la iniciación deportiva, una experiencia Cubana. ISCF Universidad Manuel Fajardo.

Weineck, Jürgen. (2005). Entrenamiento total. Primera edición, Editorial: Paidotribo. Barcelona

Wilmore, Jack H. \& Costill, David L. (2001). Fisiología del esfuerzo y del deporte. 4ta edición, Editorial Paidotribo. Barcelona 